\title{
Recent Trends in Optical Manipulation Inspired by Mesoscale Photonics and Diffraction Optics
}

\author{
Igor V. Minin ${ }^{1,2 *}$ and Oleg V. Minin ${ }^{1,2}$ \\ ${ }^{1}$ Siberian State University of Geosystems and Technologies, 10 Plahotnogo Ave., Novosibirsk 630005, Russia \\ ${ }^{2}$ Tomsk State University, 36 Lenina Ave., Tomsk 634050, Russia
}

*e-mail: prof.minin@gmail.com

\begin{abstract}
The spatial resolution of conventional optics, which is need for nondestructively trapping of microobjects, is limited by diffraction to nearly half the wavelength. Despite this limitation, the use of optical methods is one of the main directions in biological and biomedical researches, since only the use of optical methods has a minimal effect on living organisms. Quick progress in this field is based on a large extent on the development of new optical technologies and significant progress in the mesoscale photonics enabled the researches to obtain novel, previously unachievable information. Below we discussed some recent trends in optical manipulations on wavelength scale based on diffractive elements - mesoscale dielectric particles as a field localization object and classical diffractive optical elements with unusual properties. (C) 2020 Journal of Biomedical Photonics \& Engineering.
\end{abstract}

Keywords: mesoscale photonics; dielectric particle; optical force; photonic nanojet; photonic hook; particle manipulation; zone plate.

Paper \#3349 received 28 Jan 2020; revised manuscript received 24 Feb 2020; accepted for publication 27 Mar 2020; published online 20 Jun 2020. doi: 10.18287/JBPE20.06.020301.

\section{Introduction}

The possibility of non-destructively trapping and transport of individual sub-micrometer objects are highly benefit in different fields of life sciences [1-4] like microfabrication [5], chemical research [6], optics [7] and biological [8], including the precise control of single biomolecules and drug delivery to living cells. The multidisciplinary nature of optomechanical manipulation of nanoparticles has allowed bringing together seemingly unrelated fields, such as electromagnetic or/and acoustical physics, biology, paving the way for a plethora of emerging applications. The physics of optical trapping is based on the balance of the scattering and gradient optical forces. The gradient optical forces move a target along the gradient of light intensity and the scattering forces push a target in the direction of light propagation [9]. Therefore, an object is pulled to the area of stronger light intensity when the gradient forces are larger than scattering one. It could be noted that for nanoparticles having diameter less than one tenth of wavelength, optical trapping of nanoparticles is challenging due to gradient forces are proportional to the particle volume [10].
However, it is well known that the spatial resolution of conventional optics is determined only by illuminated wavelength and optical objective numerical aperture, and limited by diffraction to nearly half the wavelength. Therefore, the key problem of this achievement is the fundamental diffraction limit that prevents from focusing a light beam down to a subwavelength volume [11].

Especially in the past years, numerous optical structured beams have been proposed to optomechanically act on nanoparticles in subwavelength scale. Below we briefly consider rapidly developing methods for localizing the electromagnetic field based on mesoscale dielectric photonics. The mesoscale dielectric photonics involves the interaction of radiation with dielectric object or material structures of intermediate scale structures (Mie size parameter $q=2 \pi r / \lambda \approx(3-20) \pi[12,13]$, where $\lambda$ is illuminating wavelength and $r$ is particle radius) that are too large to be characterized as simple dipoles and too small to be described by geometrical optics.

Subwavelength confining and controlling electromagnetic energy usually involves a resonant phenomenon $[13,14]$. Below we briefly consider a class of nonresonant diffraction elements (mesoscale 
dielectric particles and unusual diffraction zone plates) for nanoparticle manipulations. In this brief review, the nexus of nanophotonics at the mesoscale and particle manipulation science is considered. This nexus offers of novel fundamental effects and interesting applications and can serve for developing groundbreaking technologies at the subwavelength scale as a starting point. Other methods of nanoparticles manipulating are widely covered in the literature and can be found by the reader in relevant reviews $[1-4,7,8,10]$.

\section{Nanohole structured dielectric mesoscale particle}

To overcome the diffraction limitation, the possibilities of light manipulation based on the mesoscale dielectric structures are becoming attractive to an increasing number of researchers. A diverse range of methods for subwavelength localization of light has been proposed. Among them, photonic crystal-based $(\mathrm{PhC})$ lenses [15-17], 3D diffractive optics [18, 19], and flat nanoslit based on the plasmonic lenses [20-22] have been studied for sharp focusing of light beyond the diffraction limit. Later, in 2017, a conical twodimensional in-plane nanoslit-based plasmonic-zone plate lens was proposed to realize far-field superresolution focusing [23] by exciting SPPs and enabling them to couple with radiating propagation modes. The graded $\mathrm{PhC}$ lenses with varying-sized air holes were studied in Refs. [24, 25]. It has been reported that by means of graded 3D PhC lens, the focused light beam with full-width at half maximum (FWHM) beam-width of about $\lambda / 75$ could be achieved [25].
However, the photonic nanojet (PNJ) phenomenon is more attractive due to the simplicity of its implementation and the compact size of the focusing particle. On the other hand, the minimum beam width of PNJ usually is about of $\lambda / 3[12,13]$ (where $\lambda$ is the wavelength of the incident light) and, thus, it is necessary to search for new methods to further reduce the size of the focal spot of the PNJ. For deep subwavelength-scale light focusing deep beyond the solid immersion diffraction limit of $\lambda / 2 n$ and strong light confinement, in Ref. [26] a nanohole-structured dielectric microsphere was proposed. The field enhancement is due to the permittivity contrast between the nanohole material and the dielectric microparticle material. The proposed nanostructured mesoscale sphere has several unique properties. For example, it could produce a high optical power and electric field intensity in low-index hole materials (air), at levels that cannot be achieved through a conventional PNJ produced by spheres without nanostructure and with the same diameter [12, 13]. It has been shown [26] that the incoming light wave is confined by the dielectric particle inside the nanohole, even when the hole diameter is deeply subwavelength (at least of $\lambda / 40$ ), obtaining a resolution near the shadow surface of the particle that is comparable to the nanohole size (beyond the solid immersion diffraction limit). The example of field localization in nanohole structured mesoscale sphere shown in Fig. 1. On the other words, the manufacturing of a nanohole on the rear surface of a dielectric mesoscale particle, allows us to "compress" the field localization characteristic of a PNJ, to the size of this nanohole.

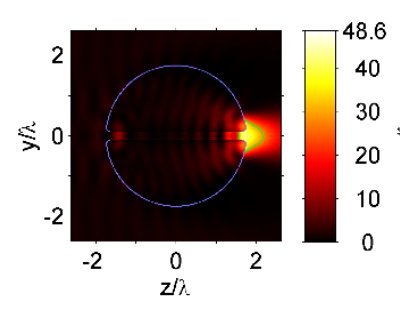

(a) hole $=\lambda / 5, z y$ plane

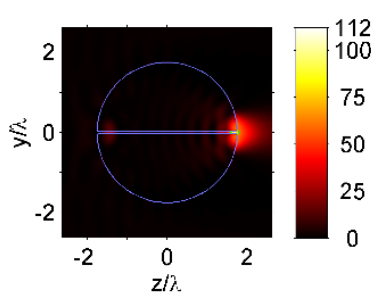

(d) hole $=\lambda / 15$, zy plane

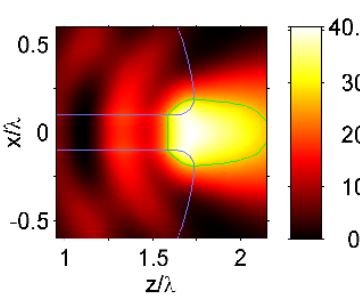

(b) focal spot, $z x$ plane

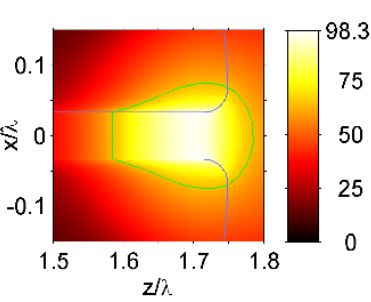

(e) focal spot, $z x$ plane

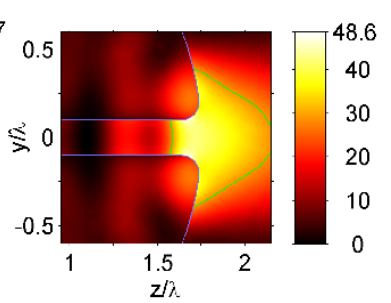

(c) focal spot, zy plane

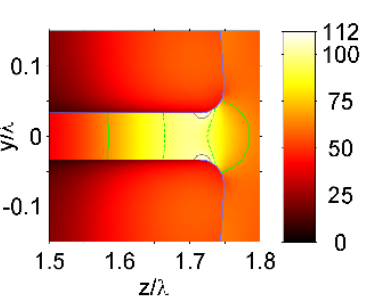

(f) focal spot, $z y$ plane

Fig. 1 Dielectric microspheres with a through hole, with the hole diameter of $(a-c)=\lambda / 5$, and $(d-f)=\lambda / 15$. The sphere diameter and refractive index are set as $D_{s}=3.5 \lambda$ and $\mathrm{n}=1.5$. Contour lines at the value of $0.5 I_{\max }, 0.85 I_{\max }$, and $0.9 I_{\max }$ are plotted with the green solid lines, green dashed lines, and gray solid lines respectively. Adapted from Ref. [26] with the permission of MDPI under a Creative Commons Attribution (CC BY) license. 

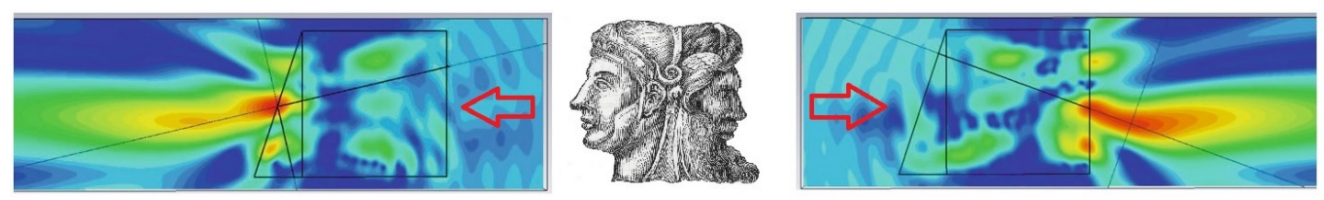

Fig. 2 The particle with broken symmetry for PH formation: the morphology of the field localization area depends on the orientation of the particle that resembles the two faces of "Janus bifrons". Adapted from Ref. [33].

The manufacture of nanohole is currently possible using one of the known methods [27-29].

Based on these investigations we propose a novel concept of "optical vacuum cleaner" [30] with simple design. The key idea is to use a designed nanoholestructured dielectric mesoscale particle of arbitrary shape for redirecting of optical momentum and achieving the desired optomechanical effects on a metallic target nanoscale object. Based on full wave numerical simulations we show that the optical forces exerted on a gold nanosphere probe are manyfold enhanced near the nanohole in mesoscale particle, that results in efficient nanoparticle propulsion into the nanohole. For example, the analysis of the phase portrait of the nanoparticle motion (focused lens with NA $=0.5$ at $\lambda=600 \mathrm{~nm}$ and with the power of $10 \mathrm{~W}$ ) has shown [30] that after a few milliseconds, the Au nanoparticle relaxes to the final point of its trajectory located approximately $20 \mathrm{~nm}$ inside dielectric cubic particle hole opening.

Nanoparticles removing from the surrounding medium is critical in many life fields, including health, air purification systems and air cabin filters [31]. Numerical simulation shows that the proposed nanohole-structured dielectric mesoscale particle can be used for optomechanical trapping and removal of metal nanoparticles, and comparing with traditional optical methods has a great potential in biomedical, chemical and many other technological applications [32].

\section{Photonic hook: a new subwavelength self-bending structured light beams}

Based on physical principles of photonic nanojet $[11,12]$ a new type of subwavelength structured light beam which called photonic hook $(\mathrm{PH})$ was discovered in 2015 [33]. Further theoretical and experimental works confirmed this general effect. Photonic hook light does not propagate along straight line but instead follow curved trajectory [33-36]. A curved beam was formed due to diffraction of an electromagnetic wave by a mesoscale dielectric Janus particle (Fig. 2) in the form of a cuboid with broken symmetry [33].

Without the wedge prism, the symmetric cuboidonly structure produced an electric-field enhancement or photonic jet, as expected, along the transmission axis. However, the addition of the wedge creates the asymmetric structure that curves the light upon exiting the cuboid to a degree dependent on the wedge angle. Wavefront analysis of such asymmetric mesoscale structure reveals that the unequal phase of the transmitted plane wave results in the irregularly concave deformation of the wavefront inside the structure that then leads to creation of the $\mathrm{PH}$ [33-38], which can be more easily visualized as a light beam with astigmatism on the macro scale. A distinctive feature of the $\mathrm{PH}$ are: the radius of curvature of $\mathrm{PH}$ is the fraction of illumination wavelength and although the curved profile evokes a similarity with Airy beams, in contrast to Airy beams there are no curved side lobes [34-37], where in the caustic from one side the sidelobes almost parallel to each other. Moreover, in the $\mathrm{PH}$ there is an inflexion where the curved beam changes its propagating direction. This property does not possessed by the Airylike beam. It is important to note that the $\mathrm{PH}$ structured field combines the construction simplicity of the PNJ, as well as the curvature produced by Airy-family selfbending beams at subwavelength level. We also note that previously studied curved beams usually require the use of expensive and complicated optical elements, which often make them unsuitable for embedding in an optical system.

Because of the phenomenon of focus bending the dielectric particle is caused by the interference of waves inside it as the phase velocity disperses the shape of the photonic hook. Also, the characteristics of curved nearfield beams [34-37] depending on the specific application can be quite exotic due to the control of phase delays across the wavefront by choosing the shape of mesoscale particles (Fig. 3).

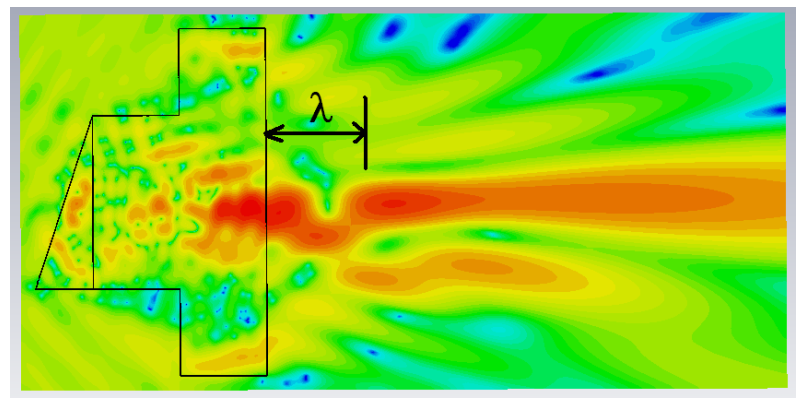

Fig. 3 Example of a photonic hook in the form of subwavelength loop.

Recent studies have investigated the generation of wide families of photonic hook beams, bringing many future opportunities to this fast-moving area including optics [33-35, 37], THz [36], surface plasmon photonics (SPP) [38], acoustics [39] and have boosted the development of mesoscale photonics providing cuttingedge abilities in particle manipulation on subwavelength scales.

The simulations of a multifocal curved beam based on $\mathrm{SiO}_{2}$ microsphere with a diameter of $433 \lambda$ (which 
does not meet the mesoscale condition and goes beyond the range of PNJ effect existence) in the optical waveband show that it is possible to control the curvature of the beam by adjusting the relative position between an off-axis Gaussian beam and a spherical particle [40]. The $\mathrm{PH}$ through a structured dielectric cylinder with embedded a glass cuboid was discussed in [41] based on numerical simulations. Furthermore, specially designed five-layer dielectric cylinder allows to produce double PHs [42]. Also, for the generation of PHs the dielectric particles with symmetry-broken material composed of materials with different refractive indexes were proposed [43, 44] (see Fig. 4) and numerically studied in Refs. [44, 45]. At the same time single light plane wave and twin-ellipse microcylinders were numerically studied to produce twin photonic hook [46].

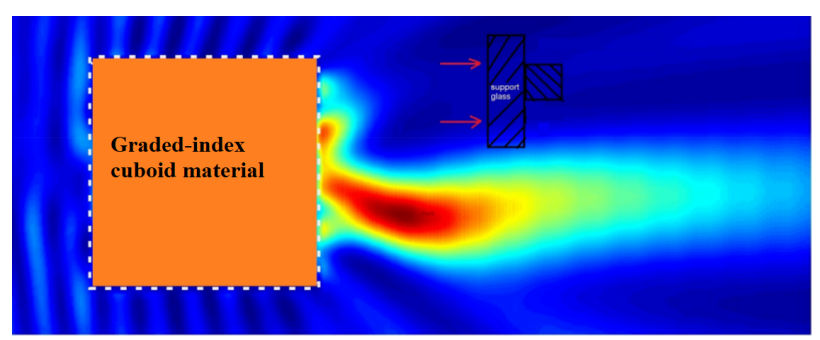

Fig. $4 \mathrm{PH}$ formation by graded-index cuboid Janus particle on substrate. Adapted from Ref. [43].

The generation of curved focus with ultrathin metalens in the visible band was recently reported in Ref. [47]. At the same time, this is not the case of our study because the lens dimensions do not satisfy the mesoscale condition formulated above. In addition, PH features are not observed and cannot be attributed to PH-type beam because its field structure possesses different spatial dependence and physical nature [3437] and strongly misleads the readers.

In general, these studies have shown the possibility to generate specialized fields that allow for realizing of more varied kinds of optical manipulation beyond trapping. In trapping studies, these investigations led to the concept of optical hook based on the optomechanical manipulator, which refers to the motion of particles on curved trajectory even around dielectric obstacles [35].

Simulations show that in the approximation that the particle is an electric dipole, i.e. Rayleigh particle [48], the target nanoparticle trajectory moves around the dielectric slab obstacle. This allows for better maneuvering of the target nanoparticles around the glass obstacles. On the other hand, the metal (Au) slab completely disrupts the field and the path trajectory of this target nanoparticle [35, 49]. One possible in vitro biomedical application of this concept is to guide the cells in a curved trajectory in order to differentiate between them [49]. The photonic hook concept offers the exquisite control over the particle's motion to manipulation and sorting of the cells in "lab-on-a-chip" platforms and microfluidic devices without the need for multiple trapping beam.

Let us note that another interesting area of application of mesoscale dielectric particles is optical traps based on the standing waves. In a standing-wave optical trap nanoparticles localized in the trap antinodes separated along the optical axis of the trap by half the working wavelength from each other. Trapping and manipulating nanoparticles in standing wave (which was generated using counter-propagating two coherent PNJs) in transmitted mode were considered in Ref. [50] and in reflection mode for the first time was investigated in Ref. [51], while the accuracy of trapping is limited by the half-wavelength period of standing waves structure. It could be noted that placing a dielectric particle with a low optical contrast on a dielectric plate with a high optical contrast makes it possible to simultaneously form two regions of field localization - two photonic jets in both reflection and transmission modes.

The applications of long PNJs for atomic cooling and trapping were considered in Ref. [52]. In this application, the Casimir-Polder potential near the surface of microparticles canceled by PNJ. The experimental confirmation of blood cells or DNA trapping and manipulation by PNJs were discussed in $[53,54]$.

\section{Non-standard modification of classical diffraction optics: particle manipulations}

The optical trapping based on the application of diffractive optical element (DOE) [55] can be classified into two main categories depending on the type of trap forces used namely vortex like beams with gradient force with orbital angular momentum (OAM) and gradient force with Gaussian beams [55]. In most optical trapping set up the gradient force of the Gaussian intensity profile [56] is used. On the other hand, the generation of vortex beam can be achieved only using complicated phase elements such as spiral phase plates which are difficult to manufacture [57].

First, we investigate the possibility of designing a spiral phase plate with only three phase levels [58]. The Poynting vector fields for the three-level spiral phase were calculated. We experimentally demonstrated an exotic three-dimensional control of structured light to show the evolution of the Poynting vector in such fields. It has been found that it possesses the orbital angular momentum suitable for optical trapping applications.

Recently, we introduce a family of simple binary DOEs to generate beams with OAM based on zone rotation principle of Fresnel zone plate with chiral focusing characteristics. As example, two different DOEs namely chiral square axicon (CSA) [59] and chiral square Fresnel zone plate (CSFZP) [60] were designed for generating structured beams with gradient force were designed as for generating structured beams with gradient force at the center with OAM surrounding it and as for only OAM for optical trapping applications. 


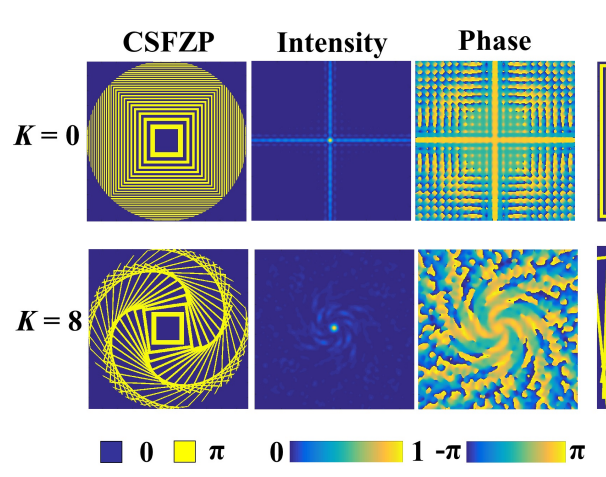

(a)

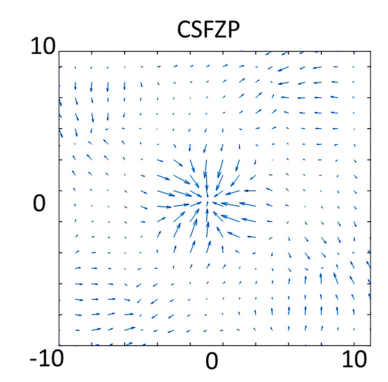

(c)

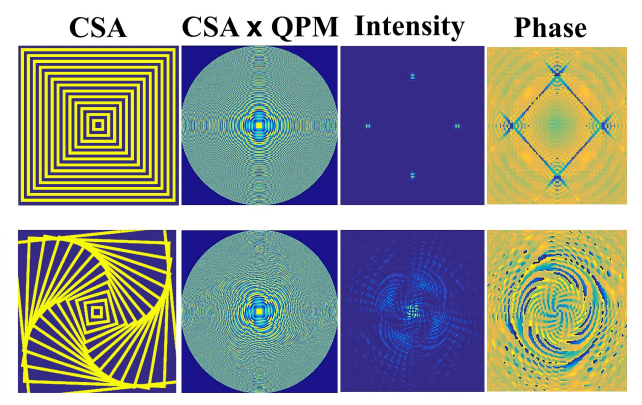

(b)

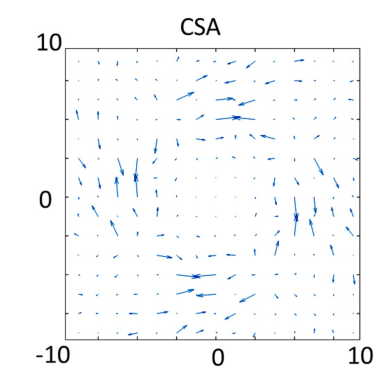

(d)

Fig. 5 Simulation of intensity and phase of (a) CSFZP and (b) CSA and Poynting vector plots for CSFZP (c) and CSA (d). The case of $K=0$ corresponds to classical DOE. Adapted from [65].
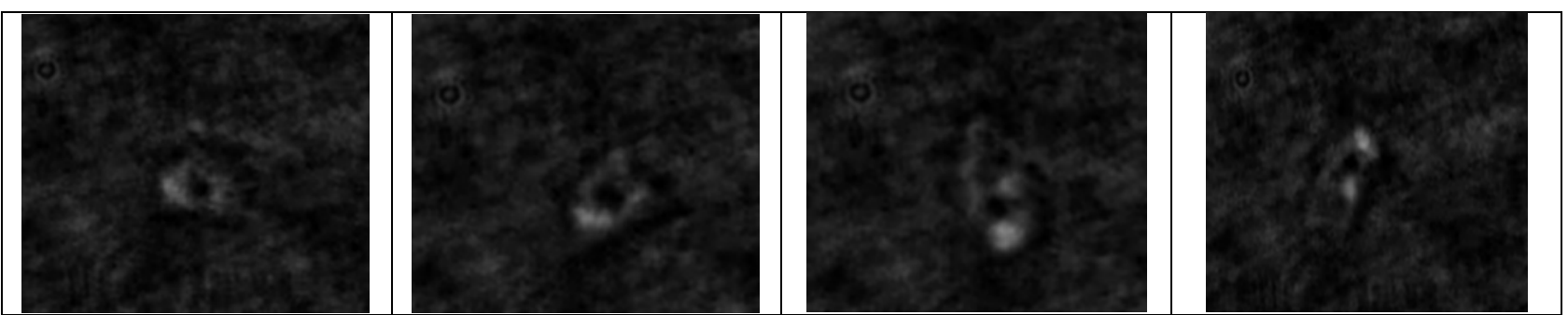

Fig. 6 Counterclockwise rotation of Candida rugose. Adapted from Ref. [59]. Experiments were performed in the laboratory of prof. C.-J. Cheng.

It could be noted that for Fresnel zone plate with square zones the boundaries of the zones do not coincide with the boundaries of the zones of the classical zone plate with circular zones [51]. Moreover, as first shown in $[18,33,62,63]$, a zone plate with a focal length less than a wavelength provides a resolution of about $\lambda / 3$.

The DOEs with binary phase values $[0, \pi]$ were designed by rotation of the half period zones with respect to one another [64].

In the case of CSA, a quadratic phase function (QPM) was multiplied to it in order to bring the far-field intensity pattern at a finite distance. The intensity and phase profiles generated by these DOEs are shown in Fig. 5. The zone rotation angle $\theta$ is $\theta=K m$, where, $m=2 n$ and $K$ is the angular integer step size. From Fig. 5 , it is followed, that with an increase of $K$ the phase twist near the optical axis showing the presence of OAM [59,60,65]. Secondly, there was a light redistribution from the focal plane of CSFZP to other axial planes that resulted an increase in the focal depth with an increase in $K$. On the other hand, in the case of CSA, with an increase in $K$ there was a light redistribution to the center creating a vortex. The Poynting vector of CSFZP shows the presence of gradient force at the center and OAM around it due to the presence of twisted side lobes and Gaussian focal spot at the center. Moreover, in CSA, only OAM is presented without gradient force at the center. Important to note that these methods do not involve any additional vortex $[0,2 \pi]$ phase elements.

The optical tweezer setup consists of two laser sources emitting at wavelengths $\lambda_{1}=532 \mathrm{~nm}$ and $\lambda_{2}=632.8 \mathrm{~nm}$ for trapping and imaging respectively. We have used Candida rugosa (ATCC $\AA 200555^{\mathrm{TM}}$ ) as a specimen for trapping and it is recorded by an imaging 
system. The sample was first trapped and then rotated by the OAM of the beam (see Figure 6).

Important to note that CBSA and CSA have some advantages compared to existing DOEs for optical trapping applications [59, 60, 65-69]. The absence of central peak in the optical field expands the applicability of CBSA to highly absorptive optical trapping specimens.

\section{Conclusion}

Mesoscale dielectric photonics has been developing rapidly lately and we can talk about the beginning of the formation of a new scientific direction - subwavelength structured field [37], despite the fact that the number of published works is extremely limited. Its distinguishing features are its small size (of the order of several wavelengths) and simple implementation. In the field of optomechanical manipulation of nanoparticles, systems based on the mesoscale photonics can be easily integrated into "lab-on-a-chip" platform. Using novel subwavelength structured light may led to the development of 'nanophotonic tweezers,' an exciting new class of 'on-chip' optical traps and can perform new bioscience. Particles can be sorted by refractive index, shape and size. Moreover, we can guide (transport) micro-particles over wavelength scaled distances for sorting sub-cellular biological material, for example, using photonic hook light or acoustical beams. Additionally, different shape of the ultraprecise laser scalpel can be designed based on the photonic hook concept [70]. The integration of a glass microsphere, produced the PNJ, on the tip of the multimode fiber [71] or Fresnel zone plate [72-74] allow to stable trapping of a single nanoparticle.

The successful realization of above briefly discussed methods can lead to fundamental changes of biomedical analysis in subwavelength scale.

\section{Disclosures}

All authors declare that there is no conflict of interests in this paper.

\section{Acknowledgence}

This work was partially supported by the Russian Foundation for Basic Research (Grant No. 20-57S52001).

\section{References}

1. K. Dholakia, T. Čižmár, "Shaping the future of manipulation," Nature Photonics 5(6), 335-342 (2011).

2. D. Gao, W. Ding, M. Nieto-Vesperinas, X. Ding, M. Rahman, T. Zhang, C. T. Lim, and C.-W. Qiu, "Optical manipulation from the microscale to the nanoscale: fundamentals, advances and prospects," Light: Science \& Applications 6(9), e17039 (2017).

3. P. Rodriguez-Sevilla, L. Labrador-Paez, D. Jaque, and P. Haro-Gonzalez, "Optical trapping for biosensing: materials and applications," Journal of Materials Chemistry B 5(46), 9085-9101 (2017).

4. D. G. Kotsifaki, S. N. Chormaic, "Plasmonic optical tweezers based on nanostructures: fundamentals, advances and prospects," Nanophotonics 8(7), 1227-1245 (2019).

5. R. Agarwal, K. Ladavac, Y. Roichman, G. H. Yu, C. M. Lieber, and D. G. Grier, "Manipulation and assembly of nanowires with holographic optical traps," Optics Express 13(22), 8906 (2005).

6. H. T. Li, D. J. Zhou, H. Browne, and D. Klenerman, "Evidence for Resonance Optical Trapping of Individual Fluorophore-Labeled Antibodies Using Single Molecule Fluorescence Spectroscopy," Journal of the American Chemical Society 128(17), 5711-5717 (2006).

7. J. R. Moffitt, Y. R. Chemla, S. B. Smith, and C. Bustamante, "Recent advances in optical tweezers," Annual Review of Biochemistry 77(1), 205-208 (2008).

8. F. M. Fazal, S. M. Block, "Optical tweezers study life under tension,” Nature Photonics 5(6), 318-321 (2011).

9. A. Ashkin, J. M. Dziedzic, J. E. Bjorkholm, and S. Chu, "Observation of a single-beam gradient force optical trap for dielectric particles," Optics Letters 11(5), 288 (1986).

10. T. Shoji, Y. Tsuboi, "Plasmonic Optical Tweezers toward Molecular Manipulation: Tailoring Plasmonic Nanostructure, Light Source, and Resonant Trapping," The Journal of Physical Chemistry Letters 5(17), 29572967 (2014).

11. E. H. K. Stelzer, "Beyond the diffraction limit?" Nature 417(6891), 806-807 (2002).

12. A. Heifetz, S. Kong, A. Sahakian, A. Taflove, and V. Backman, "Photonic nanojets," Journal of Computational and Theoretical Nanoscience 6(9), 1979-1992 (2009).

13. B. Luk'yanchuk, R. Paniagua-Domínguez, I. V. Minin, O. V. Minin, and Z. Wang, "Refractive index less than two: photonic nanojets yesterday, today and tomorrow," Optical Materials Express 7(6), 1820-1847 (2017).

14. Z. Wang, B. Luk'yanchuk, L. Yue, R. Paniagua-Domínguez, B. Yan, J. Monks, O. V. Minin, I. V. Minin, S. Huang, and A. Fedyanin, "High order Fano resonances and giant magnetic fields in dielectric microspheres," Scientific Reports 9(1), 20293 (2019). 
15. B. Wang, L. Shen, and S. He, "Superlens Formed by a One-dimensional Dielectric Photonic Crystal," Journal of the Optical Society of America B 25(3), 391-395 (2008).

16. I. V. Minin, O. V. Minin, Y. R. Triandaphilov, and V. V. Kotlyar, "Focusing properties of two types of diffractive photonic crystal lens," Optical Memory and Neural Networks 17(3), 244-248 (2008).

17. F. Gaufillet, E. Akmansoy, "Design and experimental evidence of a flat graded-index photonic crystal lens," Journal of Applied Physics 114(8), 083105 (2013).

18. I. V. Minin, O. V. Minin, N. Gagnon, and A. Petosa, "FDTD analysis of a flat diffractive optics with sub-Reyleigh limit resolution in MM/THz waveband," Proceeding of the Joint 31st International Conference on Infrared and Millimeter Waves and 14th International Conference on Terahertz Electronics, September 18-22, Shanghai, China (2006).

19. I. V. Minin, O. V. Minin, "3D diffractive lenses to overcome the 3D Abbe subwavelength diffraction," Chinese Optics Letters 12(6), 060014 (2014).

20. Y. Zhu, S. Zhou, Z. Wang, Y. Yu, W. Yuan, and W. Liu, "Investigation on Super-Resolution Focusing Performance of a TE-Polarized Nanoslit-Based Two-Dimensional Lens," Nanomaterials 10(1), 3 (2020).

21. K. R. Chen, "Focusing of light beyond the diffraction limit of half the wavelength," Optics Letters 35(22), 3763 (2010).

22. S. Ishii, A. V. Kildishev, V. M. Shalaev, and V. P. Drachev, "Controlling the wave focal structure of metallic nanoslit lenses with liquid crystals," Laser Physics Letters 8(11), 828-832 (2011).

23. R. G. Mote, O. V. Minin, and I. V. Minin, "Focusing behavior of 2-dimensional plasmonic conical zone plate," Optical and Quantum Electronics 49(8), 271 (2017).

24. L. Jin, Q. Y. Zhu, Y. Q. Fu, and W. X. Yu, "Flat lenses constructed by graded negative index-based photonic crystals with tuned configurations," Chinese Physics B 22(10), 104101 (2013).

25. Y. H. Li, Y. Q. Fu, O. V. Minin, and I. V. Minin, "Ultrasharp nanofocusing of graded index photonic crystalbased lenses perforated with optimized single defect," Optical Material Express, 6(8), 2628-2636 (2016).

26. Y. Cao, Z. Liu, O. V. Minin, and I. V. Minin, "Deep subwavelength-scale light focusing and confinement in nanohole-structured mesoscale dielectric spheres," Nanomaterials 9(2), 186 (2019).

27. T. Sakai, Y. Tanaka, Y. Nishizawa, M. Terakawa, and M. Obara, "Size parameter effect of dielectric small particle mediated nano-hole patterning on silicon wafer by femtosecond laser," Applied Physics A 99(1), 39-46 (2010).

28. J. R. Ong, H. S. Chu, V. H. Chen, A. Y. Zhu, and P. Genevet, "Freestanding dielectric nanohole array metasurface for mid-infrared wavelength applications," Optics Letters 42(13), 2639-2642 (2017).

29. Q. Wang, W. Han, Y. Wang, M. Lu, and L. Dong, "Tape nanolithography: a rapid and simple method for fabricating flexible, wearable nanophotonic devices," Microsystems \& Nanoengineering 4(1), 31 (2018).

30. I. V. Minin, O. V. Minin, Y. Cao, Z. Liu, Y. Geints, and A. Karabchevsky, "Optical vacuum cleaner by optomechanical manipulation of nanoparticles using nanostructured mesoscale dielectric cuboid," Scientific Reports 9(1), 12748 (2019)

31. C.-S. Wang, Y. Otani, "Removal of nanoparticles from gas streams by fibrous filters: a review," Industrial \& Engineering Chemistry Research 52(1), 5-17 (2013).

32. D. Erickson, X. Serey, Y. F. Chen, and S. Mandal, "Nanomanipulation using near field photonics," Lab on a Chip 11(6), 995 (2011).

33. I. V. Minin, O. V. Minin, Diffractive Optics and Nanophotonics: Resolution Below the Diffraction Limit, Springer, Cham (2016).

34. L. Yue, O. V. Minin, Z. Wang, J. Monks, A. Shalin, and I. V. Minin, "Photonic hook: a new curved light beam," Optics Letters 43(4), 771-774 (2018).

35. A. Ang, A. Karabchevsky, I. V. Minin, O. V. Minin, S. Sukhov, and A. Shalin, "Photonic Hook based optomechanical nanoparticle manipulator," Scientific Reports 8(1), 2029 (2018).

36. I. V. Minin, O. V. Minin, G. Katyba, N. Chernomyrdin, V. Kurlov, K. Zaytsev, L. Yue, Z. Wang, and D. Christodoulides, "Experimental observation of a photonic hook," Applied Physics Letters 114(3), 031105 (2019).

37. K. Dholakia, G. Bruce, “Optical hooks,” Nature Photonics 13(4), 229-230 (2019).

38. I. V. Minin, O. V. Minin, D. S. Ponomarev, and I. A. Glinskiy, "Photonic hook plasmons: a new curved surface wave," Annalen der Physik 530(12), 1800359 (2018).

39. C. Rubio, D. Tarrazó-Serrano, O. V. Minin, A. Uris, and I. V. Minin, “Acoustical hooks: a new subwavelength self-bending beam," Results in Physics 16, 102921 (2020)

40. E. Xing, H. Gao, J. Rong, S. Khew, H. Liu, C. Tong, and M. Hong, "Dynamically tunable multi-lobe laser generation via multifocal curved beam," Optics Express 26(23), 30944-30951 (2018).

41. J. Yang, P. Twardowski, P. Gérard, Y. Duo, J. Fontaine, and S. Lecler, "Ultra-narrow photonic nanojets through a glass cuboid embedded in a dielectric cylinder," Optics Express 26(4), 3723-3731 (2018).

42. Y. Huang, Z. Zhen, Y. Shen, C. Min, and G. Veronis, "Optimization of photonic nanojets generated by multilayer microcylinders with a genetic algorithm," Optics Express 27(2), 1310-1325 (2019). 
43. I. V. Minin, O. V. Minin, "Subwavelength self-bending structured light beams," Proceeding of the Fourth RussianBelarusian Workshop "Carbon nanostructures and their electromagnetic properties", April 21-24, Tomsk, 52-57 (2019).

44. I. V. Minin, O. V. Minin, "Dielectric particle-based strategy to design a new self-bending subwavelength structured light beams," Proceedings of the 14th International Forum on Strategic Technology (IFOST 2019), October 14-17, TPU Publishing House, Tomsk (2019).

45. G. Gu, L. Shao, J. Song, J. Qu, K. Zheng, X. Shen, Z. Peng, J. Hu, X. Chen, M. Chen, and Q. Wu, "Photonic hooks from Janus microcylinders," Optic Express 27(26), 37771-37780 (2019).

46. X. Shen, G. Gu, L. Shao, Z. Peng, J. Hu, S. Bandyopadhyay, Y. Liu, J. Jiang, and M. Chen, "Twin photonic hook generated by twin-ellipse microcylinder," IEEE Photonics Journal 12(3), 1-9 (2020).

47. X. Ma, Y. Guo, M. Pu, J.J. Jin, P. Gao, X. Li, and X. Luo, "Tunable Optical Hooks in the Visible Band Based on Ultra-Thin Metalenses,” Annalen der Physik 532(1), 1900396 (2019).

48. X. Cui, D. Erni, and C. Hafner, "Optical forces on metallic nanoparticles induced by a photonic nanojet," Optics Express 16(18), 13560-13568 (2008).

49. A. S. Ang, I. V. Minin, O. V. Minin, S. V. Sukhov, A. Shalin, and A. Karabchevsky, "Low-contrast photonic hook manipulator for cellular differentiation," Proceeding of the the 9th International conference on Metamaterials, Photonic crystals and Plasmonics, June 24-July 1, Marseille, France (2018).

50. H. Wang, X. Wu, and D. Shen, "Trapping and manipulating nanoparticles in photonic nanojets," Optics Letters 41(7), $1652(2016)$.

51. I. V. Minin, O. V. Minin, V. Pacheco-Peña, and M. Beruete, "Subwavelength, standing-wave optical trap based on photonic jets," Quantum Electronics 46(6), 555-557 (2016).

52. V. Yannopapas, "Photonic nanojets as three-dimensional optical atom traps: a theoretical study," Optics Communications 285(12), 2952-2955 (2012).

53. Y.-C. Li, H.-B. Xin, H.-X. Lei, L.-L. Liu, Y.-Z. Li, Y. Zhang, and B.-J. Li, "Manipulation and detection of single nanoparticles and biomolecules by a photonic nanojet," Light: Science \& Applications 5(12), e16176 (2016).

54. Y. Li, H. Xin, X. Liu, Y. Zhang, H. Lei, and B. Li, "Trapping and Detection of Nanoparticles and Cells Using a Parallel Photonic Nanojet Array," ACS Nano 10(6), 5800-5808 (2016).

55. M. Padgett, R. Bowman, "Tweezers with a twist," Nature Photonics 5(6), 343-348 (2011).

56. A. Ashkin, "Acceleration of trapping of particles by radiation pressure," Physical Review Letters 24(4), 156-159 (1970).

57. W. G. Cheong, W. M. Lee, X.-C. Yuan, L.-S. Zhang, K. Dholakia, and H. Wang, "Direct electron-beam writing of continuous spiral phase plates in negative resist with high power efficiency for optical manipulation," Applied Physics Letters 85(23), 5784-5786 (2004).

58. A. Vijayakumar, C. Rosales-Guzmán, M. R. Rai, J. Rosen, O. V. Minin, I. V. Minin, and A. Forbes, “Generation of structured light by multilevel orbital angular momentum holograms," Optics Express, 27(5), 5459 (2019).

59. B. Vinoth, A. Vijayakumar, M. Ratnam Rai, J. Rosen, C.-J. Cheng, O. V. Minin, and I. V. Minin, "Binary square axicon with chiral focusing properties for optical trapping," Optical Engineering 59(4), 041204 (2019).

60. A. Vijayakumar, B. Vinoth, I. V. Minin, J. Rosen, O. V. Minin, and C.-J. Cheng, "Experimental demonstration of square Fresnel zone plate with chiral side lobes," Applied Optics 56(13), F128-F133 (2017).

61. I. V. Minin, O. V. Minin, A. Petosa, and S. Thirakoune, "Improved zoning rule for designing square Fresnel zone plate lenses," Microwave and Optical Technology Letters 49(2), 276-278 (2007).

62. I. V. Minin, O. V. Minin, N. Gagnon, and A. Petosa, "Investigation of the resolution of phase correcting Fresnel lenses with small values of F/D and subwavelength focus," Computer optics 30, 65-68 (2006).

63. I. V. Minin, O. V. Minin, "Experimental verification 3D subwavelength resolution beyond the diffraction limit with zone plate in millimeter wave," Microwave and Optical Technology Letters 56(10), 2436-2439 (2014).

64. I. V. Minin, O. V. Minin, E. Danilov, and G. Lbov, "Parameters optimization algorithm of a new type of diffraction optics elements," Proceedings of 5th IEEE-Russia Conference MEMIA, December 13-15, Novosibirsk, Russia, 177-185 (2005).

65. A. Vijayakumar, M. R. Rai, J. Rosen, B. Vinoth, C.-J. Cheng, O. V. Minin, and I. V. Minin, "Diffractive optical elements with chiral-focusing properties for optical-trapping applications," Chapter in Frontier Research and Innovation in Optoelectronics Technology and Industry, K. Habib and E. Lewis (eds.), Tailor and Francis, London, 461-465 (2019).

66. A. Rohrbach, E. H. K. Stelzer, “Optical trapping of dielectric particles in arbitrary fields," Journal of the Optical Society of America A 18(4), 839-882 (2001).

67. J. Arlt, V. Garces-Chavez, W. Sibbett, and K. Dholakia, "Optical micromanipulation using Bessel light beam," Optics Communications 197(4-6), 239-245 (2001).

68. J. Ng, Z. Lin, and C. T. Chan, "Theory of Optical Trapping by an Optical Vortex Beam," Physical Review Letters 104(10), 103601 (2010).

69. J. Guan, J. Lin, Y. Ma, J. Tan, and P. Jin, “A subwavelength spot and a three-dimensional optical trap formed by a single planar element with azimuthal light," Scientific Reports 7(1), 7380 (2017). 
70. O. V. Minin, I. V.Minin, and N. Kharitoshin, "Microcubes Aided Photonic Jet Scalpel Tips for Potential Use in Ultraprecise Laser Surgery," Proceeding of 2015 International Conference on Biomedical Engineering and Computational Technologies (SIBIRCON), October 28-30, Novosibirsk, Russia, 18-21 (2015).

71. X. Tang, Y. Zhang, W. Su, Y. Zhang, Z. Liu, X. Yang, J. Zhang, J, Yang, and L. Yuan, "Super-low-power optical trapping of a single nanoparticle," Optics Letters 44(21), 5165 (2019).

72. A. Asadollahbaik, S. Thiele, K. Weber, A. Kumar, J. Drozella, F. Sterl, A. M. Herkommer, H. Giessen, and J. Fick, "Highly Efficient Dual-Fiber Optical Trapping with 3D Printed Diffractive Fresnel Lenses," ACS Photonics 7(1), 88-97 (2020).

73. R.S. Rodrigues Ribeiro, P. Dahal, A. Guerreiro, P. A. S. Jorge, and J. Viegas, "Fabrication of Fresnel plates on optical fibres by FIB milling for optical trapping, manipulation and detection of single cells," Scientific Reports 7, 4485 (2017).

74. L. Ma, J. Guan, Y. Wang, C. Chen, J. Zhang, J. Lin, J. Tan, and P. Jin. "Diffraction-limited axial double foci and optical traps generated by optimization-free planar lens," Nanophotonics 9(4), 841-853 (2020). 